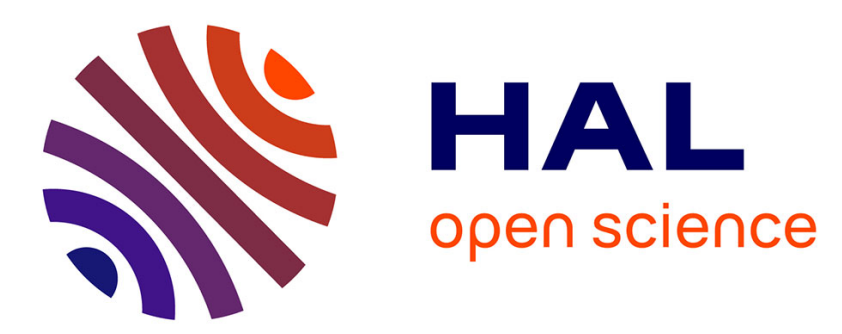

\title{
A new ONERA-CNES Slot Electron Model.
}

\author{
A. Sicart-Piet, D. Boscher, D. Lazaro, S. Bourdarie, G. Rolland
}

\section{To cite this version:}

A. Sicart-Piet, D. Boscher, D. Lazaro, S. Bourdarie, G. Rolland. A new ONERA-CNES Slot Electron Model.. RADECS 2013 (Radiation Effects on Components and Systems), Sep 2013, OXFORD, United Kingdom. hal-01057772

\section{HAL Id: hal-01057772 \\ https://hal-onera.archives-ouvertes.fr/hal-01057772}

Submitted on 25 Aug 2014

HAL is a multi-disciplinary open access archive for the deposit and dissemination of scientific research documents, whether they are published or not. The documents may come from teaching and research institutions in France or abroad, or from public or private research centers.
L'archive ouverte pluridisciplinaire $\mathbf{H A L}$, est destinée au dépôt et à la diffusion de documents scientifiques de niveau recherche, publiés ou non, émanant des établissements d'enseignement et de recherche français ou étrangers, des laboratoires publics ou privés. 


\title{
A new ONERA-CNES Slot Electron Model
}

\author{
A. Sicard-Piet ${ }^{1}$, D. Boscher ${ }^{1}$, D. Lazaro ${ }^{1}$, S. Bourdarie ${ }^{1}$, G. Rolland ${ }^{2}$ \\ 1) ONERA-The French Aerospace Lab, 2 avenue E. Belin, 31055 Toulouse, France \\ 2) Centre National d'Etudes Spatiales, 31400 Toulouse, France
}

A. Sicard-Piet is with ONERA-The French Aerospace Lab, 2 avenue E. Belin, 31055 Toulouse, France

(tel: 33-562252881, email: angelica.sicard@onera.fr).

D. Boscher is with ONERA-The French Aerospace Lab, 2 avenue E. Belin, 31055 Toulouse, France

(tel: 33-562252753, email: daniel.boscher@,onera.fr).

D. Lazaro is with ONERA-The French Aerospace Lab, 2 avenue E. Belin, 31055 Toulouse, France

(tel: 33-562252708, email: didier.lazaro@,onera.fr).

S. Bourdarie is with ONERA-The French Aerospace Lab, 2 avenue E. Belin, 31055 Toulouse, France (tel: 33-562252756, email: sebastien.bourdarie@onera.fr).

G. Rolland is with CNES, 18 av. E. Belin 31401 Toulouse, France

( tel 33-561273664: email : guy.rolland@,cnes.fr)

\begin{abstract}
A new model of electron flux in the Slot Region has been developed at ONERA. This model is based on several data sets, low altitudes data as POES or SAC-C measurements, but also data at higher altitudes as HEO1, HEO3, ICO and CRRES measurements. This model provides mean electron flux between $L=2$ and $L=4$ for energies between $0.1 \mathrm{MeV}$ and $3 \mathrm{MeV}$. This model includes a confidence level which takes into account the dynamics of electron flux in the slot region.
\end{abstract}

Index terms- Energetic particles-Slot region-Radiation belts-Specification model.

Session preference $\quad:$ Space, atmospheric and terrestrial environments

Presentation preference : Poster 


\title{
A new ONERA-CNES Slot Electron Model
}

\author{
A. Sicard-Piet, D. Boscher, D. Lazaro, S. Bourdarie, G. Rolland
}

\begin{abstract}
A new model of electron flux in the Slot Region has been developed at ONERA. This model is based on several data sets, low altitudes data as POES or SAC-C measurements, but also data at higher altitudes as HEO1, HEO3, ICO and CRRES measurements. This model provides mean electron flux between $L=2$ and $L=4$ for energies between $0.1 \mathrm{MeV}$ and $3 \mathrm{MeV}$. This model includes a confidence level which takes into account the dynamics of electron flux in the slot region.
\end{abstract}

\section{INTRODUCTION}

The Slot Region is a region of the Earth radiation belts, located between $\mathrm{L}=2$ and $\mathrm{L}=4$ nearly, in which electron flux is very low, during low magnetic activity. However, it has been observed many times an increase of this flux in slot region during strong magnetic storms [1][2][3]. Concerning the origin of the Slot Region, it now seems clear that this region of low electron flux is due to the diffusion of energetic electrons by waves during the radial diffusion of electrons from the outer radiation belt toward low L values [4] [5]. The frequencies of these waves are of the order of one hundred Hz. Several models exist and provide electron flux in the slot region [1][2][3]. Dmitriev et al. [1] try to demonstrate a dependence between the slot location, the electron flux and the interplanetary conditions, by using CORONAS-I data. Then, Fung et al. [2] studied the long term variations of the slot region with POES data. Finally, Brautigam et al. [3] used data from CEASE instrument on board TSX5 spacecraft to develop an electron flux model in the slot region and compared the results to the NASA AE8 model [6]. These three papers highlighted several interesting points such as the dependence on energy of the location of the slot region or the underestimation of electron flux from AE8 . However, some limitations appeared in these papers as for example the energy and L coverage.

Consequently, it appears essential to develop a new electron flux model, more complete and easily usable by engineers. To develop a good slot model, it is necessary to rely on data with high temporal coverage, which is the case of POES data [7], which cover more than two solar cycles, and SAC-C [8] data that represent a little more than ten years of measurements. These data are essential but only cover high latitudes and not the entire field line. However, it has been shown in previous studies a correlation between fluxes at high latitudes and at the equator in the radiation belts. Indeed, the coherence of the radiation belts of electrons was first highlighted by Kanekal et al. [9]. They showed that electron

This work was supported by grant $n^{\circ}$ R-S12/MT-0003-81 which is part of CNES R\&T program. Authors thank the PIs of all instruments whose data were used in this study.

A. Sicard-Piet, D. Boscher, D. Lazaro, S. Bourdarie are with ONERA-The French Aerospace Lab, 31055 Toulouse France (telephone; +33-562-2881, fax : +33-562-2569, e-mail: angelica.sicard@onera.fr).

G. Rolland is with CNES, 31401 Toulouse, France flux of energy $\mathrm{E}>2 \mathrm{MeV}$ measured at low altitude by SAMPEX was well correlated with measurements of the same energy range in the magnetosphere by different satellites (HEO, GOES and POLAR). This coherence was first extended to a larger energy range calculating correlations between high latitudes data with data all along the field line. To do this, we used the data from POES and SAC-C spacecraft for low altitude and any spacecraft which cut field lines inside the slot at different latitudes (HEO3, HEO1, CRRES, and ICO). From these correlations, it is then interesting to generalize the formula to correlate flux at low altitude with flux along field lines included in the region of the slot, that is to say, between $\mathrm{L}=2$ and $\mathrm{L}=4$.

\section{DATA USED}

\section{A. Low Earth Orbit Data}

At LEO orbit, we have essentially used measurements from POES spacecraft (TIROS, NOAA-06, NOAA-08, NOAA-10, NOAA-12, NOAA-14, POES-15, POES-16, POES-17, POES-18 and POES-19). To complete LEO data, measurements from ICARE detector, on board SAC-C spacecraft have been used. These electron data have been analysed and filtered in order to remove all bad measurements. Then, they have been daily averaged for the study. Table 1 resumes the characteristics of data used at LEO.

\begin{tabular}{|l|c|c|}
\hline \multicolumn{1}{|c|}{ Spacecraft } & Time coverage & Channels \\
\hline NOAA-6/SEM & $07 / 1979 \rightarrow 11 / 1986$ & \\
NOAA-8/SEM & $05 / 1983 \rightarrow 11 / 1985$ & $>100 \mathrm{keV}$ \\
NOAA-10/SEM & $10 / 1986 \rightarrow 08 / 1991$ & $>300 \mathrm{keV}$ \\
NOAA-12/SEM & $06 / 1991 \rightarrow 07 / 2002$ & $>1.1 \mathrm{MeV}$ \\
NOAA-14/SEM & $01 / 1995 \rightarrow 12 / 2004$ & \\
\hline NOAA-15/SEM2 & $07 / 1998 \rightarrow$ now & $>100 \mathrm{keV}$ \\
NOAA-16/SEM2 & $10 / 2000 \rightarrow$ now & $>300 \mathrm{keV}$ \\
NOAA-17/SEM2 & $07 / 2002 \rightarrow$ now & $\sim 3.35 \mathrm{MeV}$ \\
NOAA-18/SEM2 & $06 / 2005 \rightarrow$ now & \\
NOAA-19/SEM2 & $02 / 2009 \rightarrow$ now & $17 \mathrm{channels} \mathrm{between}$ \\
\hline SAC-C ICARE & $11 / 2000 \rightarrow$ now & $190 \mathrm{keV}$ and 3.6MeV \\
\hline
\end{tabular}

Table 1: Characteristics of data used at LEO

\section{B. Data in the magnetosphere}

For orbits in the magnetosphere intersecting the region between $\mathrm{L}=2$ and $\mathrm{L}=4$, data from 4 spacecraft have been used: CRRES [10], ICO, HEO1 and HEO3. Other spacecraft passing through this slot region exist but their data are difficult to use either because of a bad statistic or because of a lack of confidence in the data. These electron data have been analysed and filtered in order to remove all bad measurements. Then, they have been daily averaged for the study. Table 2 resumes the characteristics of data used at lower latitudes than LEO. 


\begin{tabular}{|c|c|l|}
\hline Satellite & Time coverage & \multicolumn{1}{|c|}{ Channels } \\
\hline CRRES/MEA & $07 / 1990 \rightarrow 10 / 1991$ & $\begin{array}{l}17 \text { channels between } 110 \\
\mathrm{keV} \text { and } 1.58 \mathrm{MeV}\end{array}$ \\
\hline ICO/DSU & $05 / 1994 \rightarrow 08 / 2006$ & $\begin{array}{l}3 \text { channels between } 0.95 \\
\mathrm{MeV} \text { and } 3.5 \mathrm{MeV}\end{array}$ \\
\hline HEO1/DSU & $05 / 1994 \rightarrow 08 / 2006$ & $>1.5 \mathrm{MeV}$ and $>4 \mathrm{MeV}$ \\
\hline HEO3/DSU & $11 / 1997 \rightarrow 05 / 2008$ & $>1.5 \mathrm{MeV}$ and $>3 \mathrm{MeV}$ \\
\hline
\end{tabular}

Table 2: Characteristics of data used at other orbits that LEO

\section{MODEL DEVELOPMENT}

\section{A. How to do correlations with the available data?}

The first step in the development of a slot model is to correlate POES measurements, whose time coverage is greater than 30 years, with data measured at lower latitudes on a given magnetic field line. Note that as POES data are the basis of our Slot model and that POES electron flux are integrated in energy, electron flux resulting from our slot model will be integral fluxes $\left(\mathrm{cm}^{-2} \cdot \mathrm{s}^{-1} \cdot \mathrm{sr}^{-1}\right)$. In order to correlate POES measurements with data from other satellites, it is necessary to compare similar energies. This is why the correlations were performed for energy channels similar to those of POES satellites: $>0.1,>0.3$ and $>1 \mathrm{MeV}$.

The first three energy channels of POES data allow developing a model between $0.1 \mathrm{MeV}$ and $1 \mathrm{MeV}$. In order to have better energy coverage in the model, it is necessary to use data above $1 \mathrm{MeV}$. However, given the poor statistics of $3.35 \mathrm{MeV}$ POES data, for this energy range $(\sim 3 \mathrm{MeV})$ we decided to use the data $\mathrm{HEO} 3$ as reference data for correlations. These data appear to be of good quality and have a statistical and temporal coverage more than reasonable (1997-2008).

Once these energy ranges are obtained and defined, correlations between POES measurements for energies $>0.1$, $>$ 0.3 and $>1.1 \mathrm{MeV}$, or HEO3 measurements for energy $>3$ $\mathrm{MeV}$, and the other satellites have been made. However, the slot region being relatively wide between $\mathrm{L}=2$ and $\mathrm{L}=4$, the correlations could be different between the inner boundary ( $\mathrm{L}$ $=2)$ and the outer boundary $(L=4)$ of the slot region. This is why the region was divided into 20 intervals, such as $2<\mathrm{L}$ $<2.1,2.1<\mathrm{L}<2.2, \ldots, 3.9<\mathrm{L}<4$. Correlations are thus performed on each interval $\mathrm{L}$.

In addition, a spacecraft as CRRES, for example, has an orbit such that it crosses a given field line at different latitudes, that is to say at different equatorial pitch angles $\alpha_{\text {eq }}$ $\left(\alpha_{e q}=\operatorname{asin}\left(\sqrt{ }\left(B_{e q} / B_{1}\right)\right.\right.$, with $B_{e q}$ the equatorial magnetic field and $B_{1}$ the local magnetic field). Thus, the equatorial pitch angles encountered by spacecraft vary with $\mathrm{L}$ and the pitch angle coverage is larger or smaller depending on the spacecraft. In the case of LEO as POES and SAC-C, the encountered pitch angles vary from $25^{\circ} \pm 5^{\circ}$ to $5^{\circ} \pm 2^{\circ}$ between $\mathrm{L}=2$ and $\mathrm{L}=4$. Pitch angle coverage encountered by these LEO spacecraft are not very large for a given L (maximum $5^{\circ}$ ), so a mean pitch angle for each $\mathrm{L}$ interval has been calculated and used to build the model.

Unlike spacecraft in LEO, the case of spacecraft like CRRES is more complicated. Indeed, CRRES crosses the field lines in the slot region at pitch angles between $35^{\circ}$ and $90^{\circ}$.
Consequently, for CRRES spacecraft, we divided the pitch angles encountered in three intervals and three electron flux were thus calculated: CRRES low pitch angle $\left(30^{\circ}<\alpha_{\mathrm{eq}}<50^{\circ}\right)$, CRRES medium $\left(50^{\circ}<\alpha_{\mathrm{eq}}<70^{\circ}\right)$ and CRRES high $\left(70^{\circ}<\alpha_{\mathrm{eq}}<90^{\circ}\right)$. In the cases of HEO1 and ICO, we considered a mean pitch angle for a given L. Finally, the case of HEO3 is a little more complex. There are "two branches" in the pitch angles encountered by the spacecraft, one at low latitude (high pitch angles: $\mathrm{HEO} 3$ high) and one at higher latitude (low pitch angles: HEO3 low) due to the orbit of HEO3.

This pitch angle averaging will be developed in the final paper.

\section{B. Correlations along a field line}

In this part two examples of correlation are presented on Fig. 1: correlation between electrons flux from POES and CRRES high for $\mathrm{E}>0.1 \mathrm{MeV}$ (a) and between electron flux from HEO3 and HEO1 for E $>3 \mathrm{MeV}$ (b), for given intervals in L. On each plot, the correlation coefficient between the two data sets is written as well as the line passing through the points at best (red line). These two graphs are only examples but correlations have been done for each data set, each pitch angle domain and each $\mathrm{L}$ interval. The high correlation coefficients obtained here show that the coherence highlighted by Kanekal et al. [9] can be extended to lower energies in the slot region.
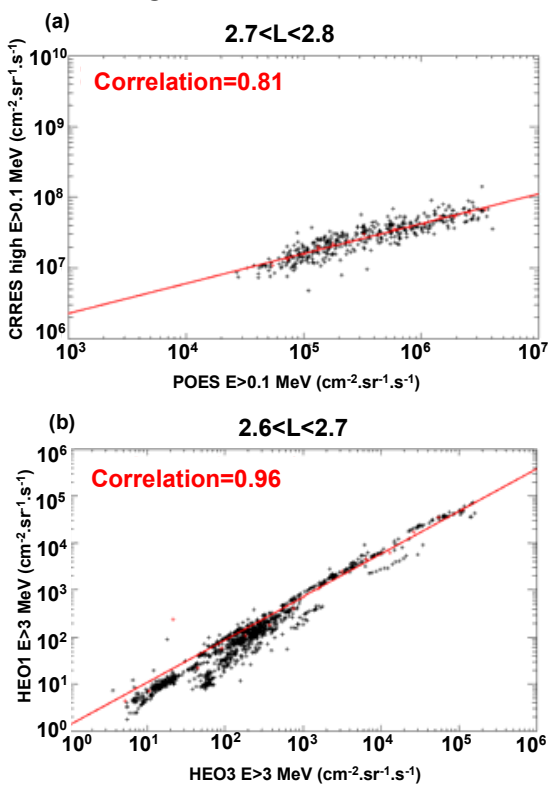

Fig. 1: Correlation between electrons flux from POES and CRRES high for $\mathrm{E}>0.1 \mathrm{MeV}$ (a) and $\mathrm{E}>0.3 \mathrm{MeV}$ (b), CRRES low for $\mathrm{E}>1 \mathrm{MeV}$ (c) and between electron flux from $\mathrm{HEO} 3$ and $\mathrm{HEO} 1$ for $\mathrm{E}>3 \mathrm{MeV}$ (d), for given intervals in $\mathrm{L}$.

\section{Variation of electron flux along magnetic field lines}

The second step in the development of the model is to calculate electron flux encountered by the spacecraft studied, from reference electron flux (POES for $\mathrm{E}>0.1, \mathrm{E}>0.3$ and $\mathrm{E}>1 \mathrm{MeV}$ and $\mathrm{HEO} 3$ for $\mathrm{E}>3 \mathrm{MeV}$ ) averaged on the lifetime of reference spacecraft and the correlation described above such as:

$$
\text { Flux }\left(L, \alpha_{\text {eq }}\right)=H\left[\operatorname{Flux}\left(L, \alpha_{\text {eq(POES or HEO3) }}\right)\right]
$$


with $H$ the correlation function (red lines in Erreur ! Source du renvoi introuvable.).

Then, the goal is to find a general equation, for each $\mathrm{L}$ interval and each energy which allow calculating mean electron flux along the magnetic field line, whatever the equatorial pitch angle. Thus, Fig. 2 presents an example of electrons flux along a field line versus equatorial pitch angle for $3.3<\mathrm{L}<3.4$, for $\mathrm{E}>1 \mathrm{MeV}$ calculated for each spacecraft by using reference data and the correlation function. The red line, whose equation is of the form $\mathrm{Y}=\exp \left(\mathrm{A} \cdot \sin (\mathrm{X})^{\mathrm{B}}\right)$, passes through the points at best.

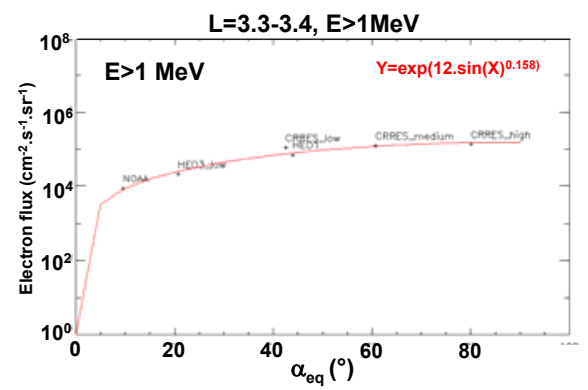

Fig. 2: Example of electron flux along a field line versus equatorial pitch angle for $3.3<\mathrm{L}<3.4$ for each spacecraft (POES flux*Correlation) for $\mathrm{E}>1$ $\mathrm{MeV}$.
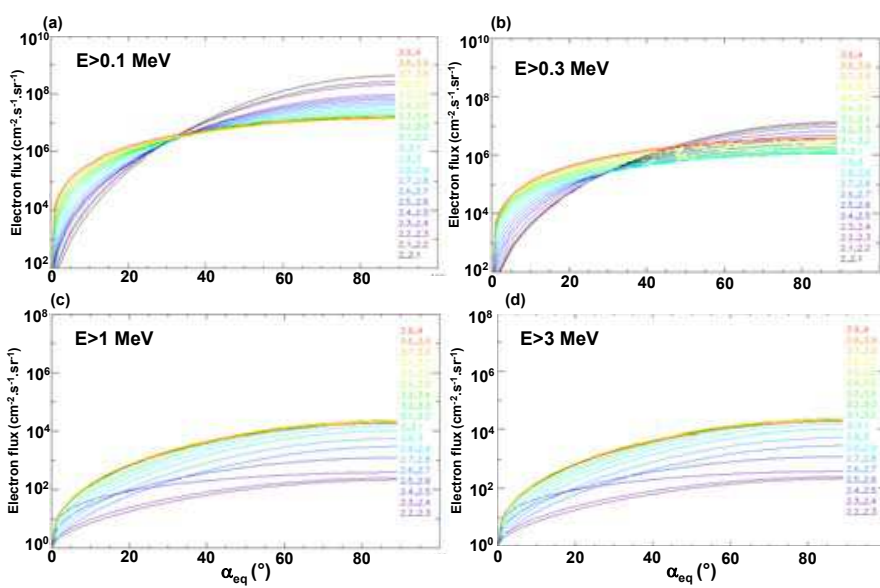

Fig. 3: Electron flux along a field line versus equatorial pitch angle for $E>0.1$ $\mathrm{MeV}$ (a), $\mathrm{E}>0.3 \mathrm{MeV}$ (b), $\mathrm{E}>1 \mathrm{MeV}$ (c) and $\mathrm{E}>3 \mathrm{MeV}$ (d) for each $\mathrm{L}$ interval.

Then Fig. 3 represents electron flux versus equatorial pitch angle for each L interval and each energy ( $\mathrm{E}>0.1 \mathrm{MeV}$ (a), $\mathrm{E}>0.3 \mathrm{MeV}$ (b), E>1 MeV (c) and E>3 MeV (d). This graphs show that two trends are emerging about the evolution of electron flux versus equatorial pitch angle according to energy. For E $>0.1 \mathrm{MeV}$ (Fig. 3 (a)), electron fluxes increase with $\mathrm{L}$ for small pitch angle $\left(\alpha_{\mathrm{eq}}<35^{\circ}\right)$ while they decrease with $\mathrm{L}$ for high pitch angle $\left(\alpha_{\mathrm{eq}}>35^{\circ}\right)$. Actually, this plot shows that, at this energy, the slot region is divided into two sub-regions, $\mathrm{L}<3$ and $\mathrm{L}>3$. For $\mathrm{L}>3$, the interaction between energetic particles and chorus waves is the main physical process and the pitch angle diffusion coefficients due to this interaction are very high and tend to make isotropic flux, while $\mathrm{L}<3$ is dominated by the radial diffusion whose effect on flux is different. For $\mathrm{E}>0.3 \mathrm{MeV}$, the tendency is the same. The profile is very different for the two higher energies ( $\mathrm{E}>1 \mathrm{MeV}$ and $\mathrm{E}>3 \mathrm{MeV}$ ) since electron fluxes increase with $\mathrm{L}$ whatever the equatorial pitch angle, excepted for the very low $\mathrm{L}$ values $(\mathrm{L}>2.5)$ for which the statistics of data is very bad. For these high energies, pitch angle diffusion coefficients due to wave particle interaction are lower so that the major physical process is the radial diffusion even for $\mathrm{L}>3$.

\section{Mean electron model in Slot region}

In summarizing the previous parts, we have developed an electron flux model in the slot region, depending on two parameters: $\mathrm{L}$ and $\alpha_{\text {eq. }}$. This model gives mean omnidirectional electron flux integrated in energy $\left(\mathrm{cm}^{-2} \cdot \mathrm{s}^{-1} \cdot \mathrm{sr}^{-}\right.$ ${ }^{1}$ ) and is valid for energies between $0.1 \mathrm{MeV}$ and $3 \mathrm{MeV}$. Fig. 4 presents an example of electron flux spectra provided by the model for several pitch angle at $L=2.5$. This graph shows that the differences between equatorial electron flux and high latitude flux is higher at $0.1 \mathrm{MeV}$ (factor $10^{4}$ ) than at $3 \mathrm{MeV}$ (factor 10).

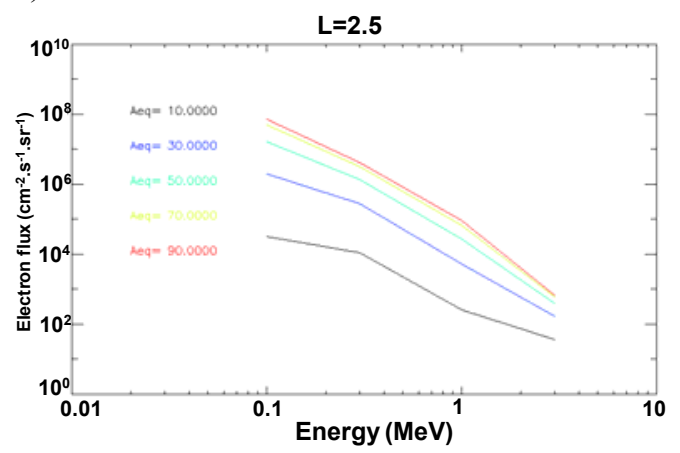

Fig. 4: Mean electron flux versus energy provided by the model at $\mathrm{L}=2.5$ and for several equatorial pitch angles

\section{COMPARISON WITH AE8 NASA MODEL}

In this section, we compare the flux obtained with our Slot model developed here and the flux obtained with the NASA AE8 model. Fig. 5 shows comparisons between fluxes obtained with our model and AE8 fluxes for two different altitudes: $8000 \mathrm{~km}$ (on top) and $16000 \mathrm{~km}$ (at bottom). The black line in solid line represents the mean flux from our model and the red curves correspond to flux from AE8 MAX (solid line) and AE8 MIN (dashed line). This figure shows that electron fluxes from our model are generally stronger than AE8 fluxes, which confirms that AE8 underestimates very significantly electron flux in the slot region, as already demonstrated in previous studies [3]. The difference between the electron mean flux from our model and AE8 results is greater at $\mathrm{L}=2.3$ (top), ranging from a factor of 10 to a factor of almost 100 , than at $\mathrm{L}=3.5$ (bottom) where the difference reached a factor up to 10 at $0.1 \mathrm{MeV}$.

This mean Slot model, presented here has been improved by taking into account the dynamics of the reference data (NPOES and HEO3) data. Thus a model with confidence level has been developed. This part of the model will be described in the final paper. This ONERA-CNES Slot model 
with confidence level has also been compared with the AE8 NASA model.
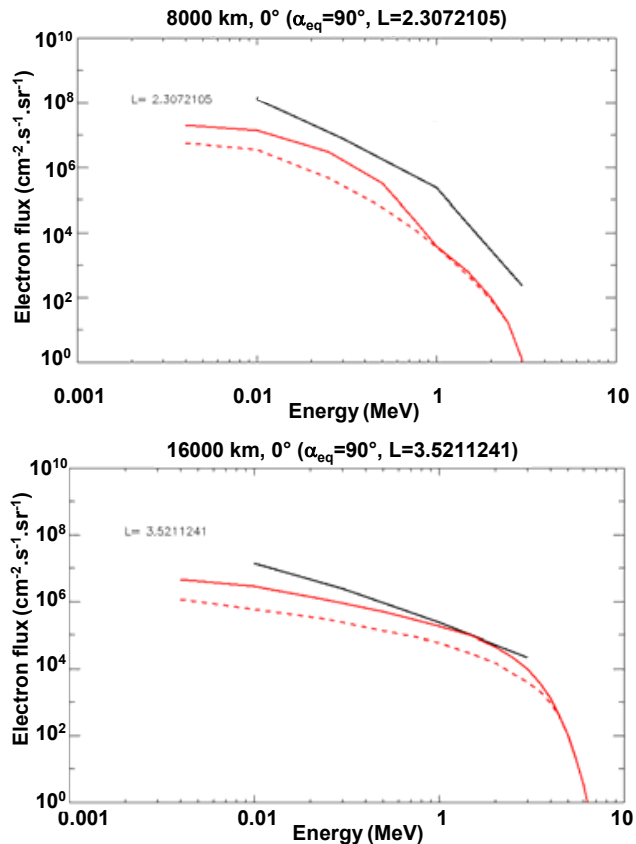

Fig. 5:Comparison between fluxes obtained with our model and AE8 model for two different altitudes: $8000 \mathrm{~km}$ (on top) and $16000 \mathrm{~km}$ (at bottom). Black solid line is the mean electron flux from our model. Red lines correspond to AE8 fluxes for AE8 MAX (solid red line) and AE8 MIN (dashed red line).

\section{DISCUSSIONS AND CONCLUSIONS}

ONERA has developed a model of electron flux in the slot region between $\mathrm{L}=2$ and $\mathrm{L}=4$, calculating integrated omnidirectional flux for energies between 0.1 and $3 \mathrm{MeV}$, using POES and HEO3 data as reference data but also CRRES, ICO, HEO1 an SAC-C data. This first version of the model has two input parameters: $\mathrm{L}, \alpha_{\mathrm{eq}}$ and gives the mean electron flux in the Slot region, between $\mathrm{L}=2$ and $\mathrm{L}=4$. This model has been improved with a confidence level, taking into account the dynamics of the data, which is not presented here but will be included in the final paper. This ONERA-CNES Slot model will be implemented soon in OMERE tool, in order to use it as easy as possible. The comparison between ONERA-CNES mean Slot model with AE8 NASA model shows that AE8 underestimates electron flux in the slot region.

\section{REFERENCES}

[1] A. V. Dmitriev, Y. S. Minaeva and Y. V. Orlov, Model of the slot region of Earth's electron radiation belts depending on the heliospheric parameters, Adv. Space Res., vol 25, No 12, 2000.

[2] S. F. Fung, X. Shao and L. C. Tan, Long-term variations of the electron slot region and global radiation belt structure, Geophys. Res. Lett., vol 33, L04105, doi:10.1029/2005GL024891, 2006.

[3] D. H. Brautigam, K; P. Ray, G. P. Ginet and D. Madden, Specification of the radiation belt slot region: comparison of the NASA AE8 model with TSX5/CEASE data, IEEE Trans. On Nuc. Sci., vol 51, No6, 2004.

[4] R. M. Thorne, Y. Y. Shprits, N. P. Meredith, R. B Horne, W. Li and L. $\mathrm{R}$. Lyons, refilling of the slot region between the inner and outer radiation belts during geomagnetic storms, J. Geophys. Res., vol 112, A06203, doi:10.1029/2006JA012176, 2007.

[5] N. P. Meredith, R. B. Horne, S; Glauert and R. R. Anderson, Slot region electron loss timescale due to plasmaspheric hiss and lightninggenerated whistlers, J. Geophys. Res., vol 112, A08214, doi:10.1029:2007JA012413, 2007.

[6] J. I. Vette, The AE8 trapped electron model environment, NSSDC/WDC-A-R \& S, National Space Science Data Center, 1991.

[7] D.S. Evans, M.S. Greer, Polar Orbiting Environmental Satellite Space Environment Monitor - 2: Instrument Descriptions and Archive Data Documentation, NOAA Technical Memorandum, Space Environment Center, Boulder, 2000

[8] D. Falguère, D. Boscher, T. Nuns, S. Duzellier, S. Bourdarie, R. Ecoffet, S. Barde, J. Cueto, C. Alonzo, and C. Hoffman, "In-flight observations of the radiation environment and its effects on devices in the SAC-C polar orbit," IEEE Trans. Nucl. Sci., vol. 49, no. 6, pp. 2782-2787, Dec. 2002.

[9] S. G. Kanekal, D. N. Baker, J. B. Blake, Multisatellite measurements of relativistic electrons: Global coherence, J; Geophys. Res., 106-A12, pp29721-29732, 2001.

[10] A.L. Vampola, The ESA outer zone electron model update, in Environment Modelling for Space-Based Applications, Symposium Proceedings (ESA SP-392), ed. by W. Burke, T.-D. Guyenne, 18-20 September 1996 (ESTEC, Noordwijk, 1996), p. 151. 